\section{Sung Shik Kang ${ }^{1}$, Hee Seok Jeong', Hee Young Son ${ }^{3}$, Tae Yong Moon ${ }^{2}$}

Departments of ${ }^{1}$ Orthopedics and ${ }^{2}$ Radiology, Pusan National University Yangsan Hospital, Yangsan, ${ }^{3}$ Department of Otorhinolaryngology, Dongnam Institute of Radiological \& Medical Sciences, Busan, Korea

\section{Corresponding Author:}

Hee Seok Jeong

Department of Radiology,

Pusan National University

Yangsan Hospital, 20, Geumo-ro,

Mulgeum-eup, Yangsan 50612, Korea

Tel: +82-55-360-1845

Fax: +82-55-360-1848

E-mail: mediknight@hanmail.net

Received: September 5, 2017

Revised: September 22, 2017

Accepted: September 28, 2017

Copyright (C) 2017 by The Korean Spinal Neurosurgery Society

This is an open access article distributed under the terms of the Creative Commons Attribution Non-Commercial License (http://creativecommons.org/licenses/bync/4.0/) which permits unrestricted noncommercial use, distribution, and reproduction in any medium, provided the original work is properly cited.

\title{
Glomangiopericytoma of the Spinal Cord
}

Glomangiopericytoma, also referred to as a hemangiopericytoma-like tumor or sinonasal type hemangiopericytoma, is a rare tumor of the nasal cavity and paranasal sinus that has never been reported in the spinal cord. Here, we report a case of a 47-year-old man who developed glomangiopericytoma in the thoracic spine. This lesion showed a heterogeneous high signal intensity compared to the spinal cord on a T2-weighted image and an easily enhanced isointense signal on a T1-weighted image.

Key Words: Glomangiopericytoma, Thoracic spine, Tumor, Spinal cord

\section{INTRODUCTION}

Glomangiopericytoma is a rare sinonasal tumor with a perivascular myoid phenotype and a prominent perivascular growth pattern ${ }^{15)}$. It develops in the nasal cavity and paranasal sinus and has a low malignant potential ${ }^{7}$. Glomangiopericytomas are different from conventional hemangiopericytomas with respect to their location and histopathologic features and account for less than $0.5 \%$ of all sinonasal tumors ${ }^{16}$. While their etiology is unclear, a history of trauma, hypertension, pregnancy, or corticosteroid use can be a cause ${ }^{1)}$. To date, there have been no reported cases of glomangiopericytoma in the spine. Here, we present what we believe to be the first reported case of spinal glomangiopericytoma, and we describe its radiological characteristics. This study was approved by the Institutional Review Board of Pusan National University Yangsan Hospital, and the need for informed consent was waived owing to the retrospective nature of the study.

\section{CASE REPORT}

A 47-year-old man was admitted for gait disturbance, radiating pain, and decreased sensation in the left calf that had developed about 15 days ago. Muscle weakness in the lower leg was not observed in the physical examination. Numbness and a tingling sensation in the left calf and foot were confirmed, and the sacral segment showed a sparing pattern. The patient had experienced frequent urination and a residual urine sensation for several months without signs of benign prostatic hypertrophy. Electrodiagnosis revealed no definite evidence of neuropathy or radiculopathy in the lower extremities, but a partial defect in neural conductivity through the posterior column-medial lemniscus system from the left lower limb was suspected. Tests for systemic tumor markers revealed that alpha-fetoprotein $(3.95 \mathrm{ng} / \mathrm{mL})$, carcinoembryonic antigen $(2.51 \mathrm{ng} / \mathrm{mL})$, and prostate-specific antigen $(0.471 \mathrm{ng} / \mathrm{mL})$ were all within the normal range. Cerebrospinal fluid (CSF) analysis revealed that the proportion of lymphocytes had increased to $100 \%$ (normal range, 28\%-96\%) and the protein level had increased to $491 \mathrm{mg} / \mathrm{dL}$ (normal range, $15-45 \mathrm{mg} / \mathrm{dL}$ ).

There were no particular findings on the plain radiograph of the thoracic and lumbar spine besides spondylosis. Magnetic resonance imaging (MRI) of the thoracic spine revealed a lobulated mass measuring approximately 1.6 $\mathrm{cm} \times 1.0 \mathrm{~cm} \times 1.5 \mathrm{~cm}$ in the left posterolateral aspect of the intramedullary area at the T12 level, and swelling and edema were found from T4 to L1 in the spinal cord. Destruction of the adjacent bone was not observed. Compared with the spinal cord, heterogeneous high-signal intensity was observed on a T2-weighted image (T2WI), an isointense signal was apparent on a T1-weighted image (T1WI), and homogeneous enhancement was detected on a contrast-enhanced T1WI. Compared with the spinal cord, an isointense lesion with definite boundaries was observed on b400 and b800 diffusion wei- ghted imaging (DWI) and in an apparent diffusion coefficient map. On nonenhanced computed tomography (CT) images, compared with the spinal cord, the mass was seen as an isodense soft tissue lesion with indefinite boundaries, and homogeneous enhancement was detected on contrast-enhanced CT. Minimal fluorodeoxyglucose (FDG) uptake 

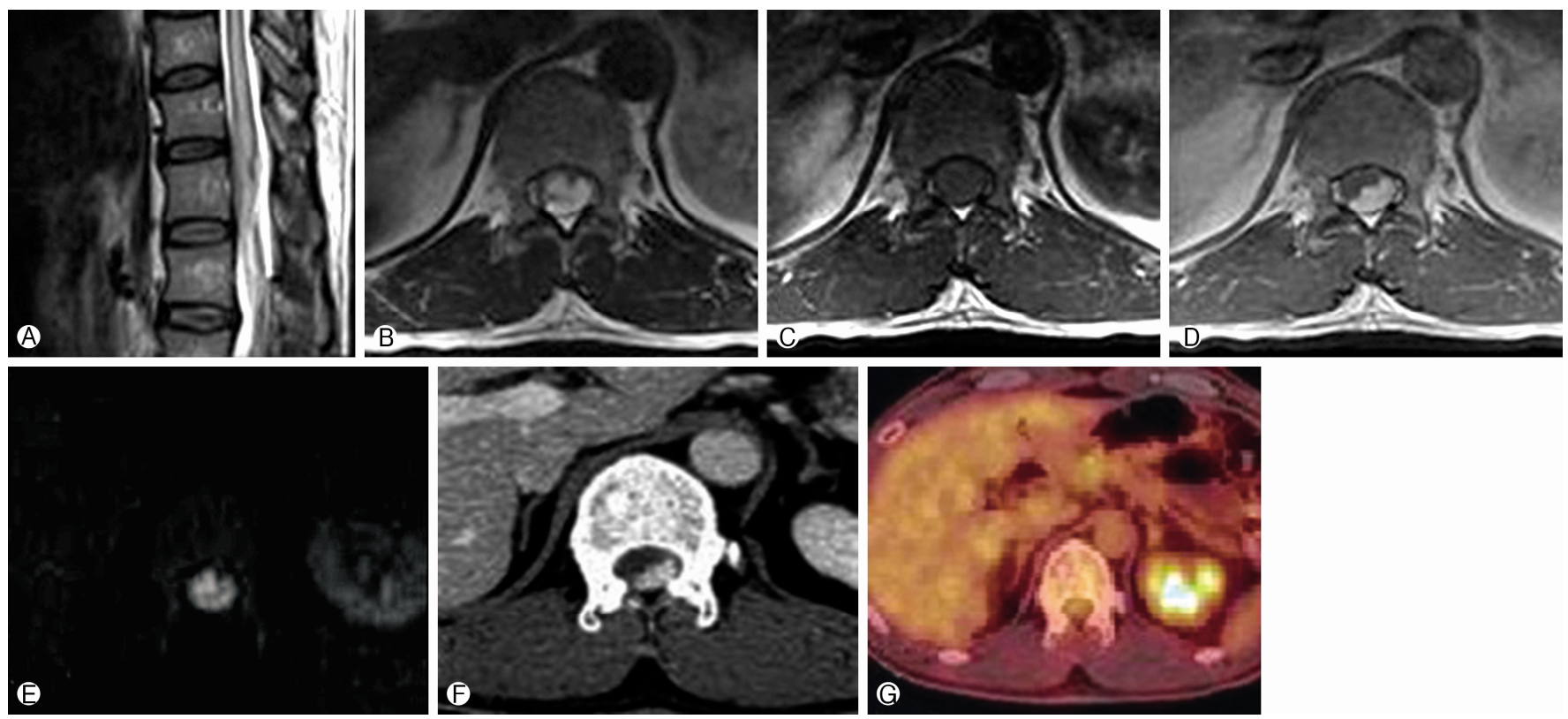

Fig. 1. Sagittal T2-weighted (A) and axial T2-weighted (B) magnetic resonance imaging revealing swelling and edema in the spinal cord. (C) T1-weighted image showing an isointense signal. (D) Postcontrast T1-weighted image with fat suppression showing a homogeneously enhanced lobulated mass. (E) Axial diffusion-weighted imaging (b800) showing an isointense signal (relative to the spinal cord) with definite lesion boundaries. $(\mathrm{F})$ Axial contrast-enhanced computed tomography (CT) images show a homogeneous enhancing mass. (G) Axial positron emission tomography-CT revealing low-grade hypermetabolism (maxSUV: 2.8) in the mass lesion.

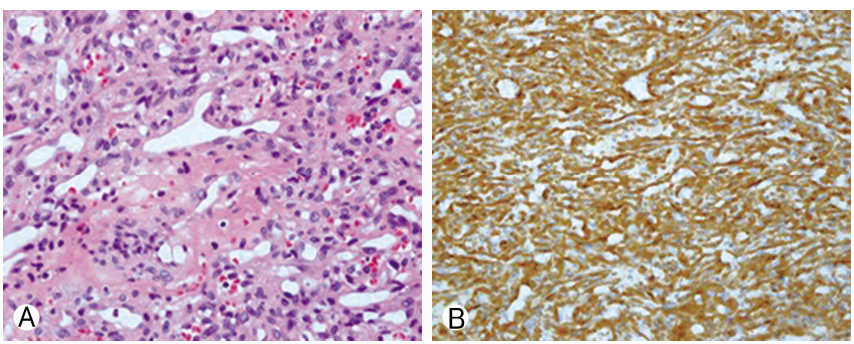

Fig. 2. (A) Hematoxylin and eosin staining $(\times 400)$ revealing numerous thin-walled vessels surrounded by oval- to spindle-shaped cells. (B) Immunohistochemical analysis revealing positive staining for smooth muscle actin $(\times 200)$

(maxSUV: 2.8) by the mass lesion was observed on positron emission tomography (PET)-CT (Fig. 1).

Radiologically, we did not distinguish between intradural extramedullary tumor or intramedullary tumor and a midline durotomy was performed following total laminectomy of T12 and left-sided partial laminectomy of L1. No tumor was detected in the intradural extramedullary space. After myelotomy, a brownish soft mass was observed that had adhered to the rootlets in the intramedullary space. Intralesional biopsy and debulking surgery were performed owing to a very irregular margin. Complete excision was impossible due to a very ill-defined margin. Furthermore, malignancy could not be ruled out. There were no perioperative and postoperative complications. The mass was a highly vascularized lesion. Bleeding control during surgery was very difficult.

Upon histological examination, hematoxylin and eosin (H\&E) staining revealed probable glomangiopericytoma cells that were oval to spindle-shaped and located in a thin-walled staghorn vessel. Immunohistochemically, positive staining for smooth muscle actin (SMA) and bcl2, but negative staining for CD31, CD34, CD99, S100, factor VIII, and epithelial membrane antigen were present in tumor cells (Fig. 2). These features confirmed the diagnosis of glomangiopericytoma.

\section{DISCUSSION}

Glomangiopericytoma was first reported in 1942 as hemangiopericytoma by Stout and Murray ${ }^{12)}$. In 1976, Compagno and Hyams ${ }^{3)}$ renamed it hemangiopericytoma-like intranasal tumor in order to reflect its low metastasis and mortality rates. In 2005, the World Health Organization named it glomangiopericytoma, sinonasal-type hemangiopericytoma, or hemangiopericytomalike tumor due to its different clinical and histological characteristics compared with hemangiopericytoma ${ }^{16}$. Glomangiopericytoma mainly develops in the nasal cavity and paranasal sinuses and accounts for less than $0.5 \%$ of all sinonasal tumors ${ }^{16}$. It is slightly more common in women than men and can occur in patients of any age, but is most common in the seventh decade of life ${ }^{2,7)}$. However, to the best of our knowledge, a case of glomangiopericytoma in the spine has never previously been reported. There was no history of trauma, hypertension, or corti- 
costeroid use in our patients and etiology was unclear. So, we think that glomangiopericytoma is considered as a hybrid between hemangiopericytoma and glomus tumor ${ }^{10)}$, it may be related to the development of hemangiopericytoma and glomus tumor, although it is rare in spine.

Glomangiopericytoma in the nasal cavity is characterized histologically by the presence of round, glomus-like cells located near the blood vessels. In H\&E staining, varying numbers of vascular channels and perivascular concentric cellular growths are seen. Nuclear pleomorphism is not observed, and there are few or no mitotic bodies ${ }^{13)}$. Immunohistochemically, these tumors are positive for vimentin, SMA, muscle specific actin, and factor XIIIa and negative for desmin, CD31, CD34, CD99, CD117, S100 protein, and bcl-2, although they have been weakly positive for CD34 and S100 protein in some cases ${ }^{5,13,15,17,18)}$. It shows soft tissue density and heterogeneous enhancement on $\mathrm{CT}^{6,9,13,14)}$. On MRI, an isointense signal (relative to normal nasal mucosa) is observed on T1WI, and an isointense-to-high signal is seen on T2WI. On contrast-enhanced MRI, unlike on CT, it shows homogeneous enhancement ${ }^{9,13)}$. In general, these tumors unilaterally involve the nasal cavity, and destruction of the neighboring bone is not observed ${ }^{6,9,13,14)}$. In PET-CT, low-grade FDG hypermetabolism is seen ${ }^{4)}$.

In the case described here, a lobulated mass was observed in the left posterolateral aspect of the intramedullary area at the T12 level of the thoracic spine, and swelling and edema were detected from T4 to L1 in the spinal cord. Similar to glomangiopericytoma in the nasal cavity, the lesion was seen as an isodense soft tissue lesion with indefinite boundaries on nonenhanced CT compared with the spinal cord, while homogeneous enhancement was observed on contrast-enhanced CT. Minimal FDG uptake was detected on PET-CT. Compared with the spinal cord on MRI, heterogeneous high-signal intensity on T2WI and an isointense signal on T1WI were observed. Like contrast-enhanced CT, homogeneous enhancement was seen on contrast-enhanced T1WI. DWI revealed an isointense signal compared with CSF, and the lesion had definite boundaries in b400 and b800. During imaging studies, it is necessary to distinguish glomangiopericytoma from astrocytoma and hemangiopericytoma that can develop in the intradural area. Histologically, fibrillary astrocytoma is seen parenchymal infiltration, nuclear atypia and increased cellularity. Pilocytic astrocytoma lacks mitotic figures and shows harbor Rosenthal fibers and thickened vascular walls ${ }^{11)}$. Hemangiopericytoma is seen loss of architecture, high cellularity with frequent mitoses, nuclear pleomorphism and staghorn' vascular channels. Immunohistochemically, these tumor can be positively for HLA-DR, CD34, Leu-7, vimentin, S-100 protein, CD99, bcl-2, and factor XIIIa ${ }^{8)}$.

\section{CONCLUSION}

Glomangiopericytoma is a rare tumor of the nasal cavity and paranasal sinus, and this is the first reported case of glomangiopericytoma in the spinal cord. Compared to glomangioper- icytoma in the nasal cavity, this case of spinal glomangiopericytoma showed very similar CT, MRI, and PET-CT findings, and no destruction of the neighboring bone was observed.

\section{CONFLICT OF INTEREST}

No potential conflict of interest relevant to this article was reported.

\section{REFERENCES}

1. Angouridakis N, Zaraboukas T, Vital J, Vital V: Sinonasal hemangiopericytoma of the middle turbinate: a case report and brief review of the literature. B-ENT 3:139-143, 2007

2. Compagno J: Hemangiopericytoma-like tumors of the nasal cavity: a comparison with hemangiopericytoma of soft tissues. Laryngoscope 88:460-469, 1978

3. Compagno J, Hyams VJ: Hemangiopericytoma-like intranasal tumors. A clinicopathologic study of 23 cases. Am J Clin Pathol 66: 672-683, 1976

4. Conrad GR, Sinha P, Absher KJ: FDG PET/CT findings of a glomangiopericytoma. Clin Nucl Med 36:462-464, 2011

5. Eichhorn JH, Dickersin GR, Bhan AK, Goodman ML: Sinonasal hemangiopericytoma. A reassessment with electron microscopy, immunohistochemistry, and long-term follow-up. Am J Surg Pathol 14:856-866, 1990

6. Higashi K, Nakaya K, Watanabe M, Ikeda R, Suzuki T, Oshima T, et al: Glomangiopericytoma of the nasal cavity. Auris Nasus Larynx 38:415-417, 2011

7. Li XQ, Hisaoka M, Morio T, Hashimoto H: Intranasal pericytic tumors (glomus tumor and sinonasal hemangiopericytoma-like tumor): report of two cases with review of the literature. Pathol Int 53:303-308, 2003

8. Liu HG, Yang AC, Chen N, Yang J, Qiu XG, Zhang JG: Hemangiopericytomas in the spine: clinical features, classification, treatment, and long-term follow-up in 26 patients. Neurosurgery 72:16-24, 2013

9. Oosthuizen JC, Kennedy S, Timon C: Glomangiopericytoma (sinonasal-type haemangiopericytoma). J Laryngol Otol 126:1069-1072, 2012

10. Rosai J: Ackerman's surgical pathology, ed 8. St. Louis (MO): Mosby, pp289-313, 1996

11. Smith AB, Soderlund KA, Rushing EJ, Smirniotopolous JG: Radiologic-pathologic correlation of pediatric and adolescent spinal neoplasms: Part 1, Intramedullary spinal neoplasms. AJR Am J Roentgenol 198:34-43, 2012

12. Stout AP, Murray MR: Hemangiopericytoma: a vascular tumor featuring Zimmermann's pericytes. Ann Surg 116:26-33, 1942

13. Sun Q, Zhang C, Chen W, He Y: The molecular mechanisms on glomangiopericytoma invasion. Orphanet J Rare Dis 8:152, 2013

14. Taglialatela Scafati C, D'Antonio A, Taglialatela Scafati S, Scotto di Clemente S, Parascandolo S: Glomangiopericytoma of the pterygomandibular space: an unusual case. Br J Oral Maxillofac Surg 45:673-675, 2007

15. Thompson LD, Fanburg-Smith JC, Wenig BM: Borderline and Low Malignant Potential Tumours of Soft Tissue in Barnes L, Eveson 
JW, Reichart P, Sidransky D (eds): World Health Organization Classification of Tumours: Pathology and Genetics of Head and Neck Tumours. Lyon (France): IARC Press, pp43-44, 2005

16. Thompson LD, Miettinen M, Wenig BM: Sinonasal-type hemangiopericytoma: a clinicopathologic and immunophenotypic analysis of 104 cases showing perivascular myoid differentiation. Am J Surg Pathol 27:737-749, 2003
17. Tse LL, Chan JK: Sinonasal haemangiopericytoma-like tumour: a sinonasal glomus tumour or a haemangiopericytoma? Histopathology 40:510-517, 2002

18. Watanabe K, Saito A, Suzuki M, Yamanobe S, Suzuki T: True hemangiopericytoma of the nasal cavity. Arch Pathol Lab Med 125:686-690, 2001 